\title{
Stochastic semiclassical cosmological models
}

\author{
Esteban Calzetta \\ Instituto de Astronomía y Física del Espacio (IAFE) and Departamento de Física, Universidad de Buenos Aires, Ciudad Universitaria, \\ 1428 Buenos Aires, Argentina \\ Antonio Campos \\ Grup de Física Teòrica and Institut de Física d'Altes Energies (IFAE), Universitat Autònoma de Barcelona, \\ 08193 Bellaterra (Barcelona), Spain \\ and Department of Physics, University of Maryland, College Park, Maryland 20742 \\ Enric Verdaguer \\ Departament de Física Fonamental and IFAE, Universitat de Barcelona, Avinguda Diagonal 647, 08028 Barcelona, Spain
}

(Received 21 March 1997)

\begin{abstract}
We consider the classical stochastic fluctuations of spacetime geometry induced by quantum fluctuations of massless nonconformal matter fields in the early Universe. To this end, we supplement the stress-energy tensor of these fields with a stochastic part, which is computed along the lines of the Feynman-Vernon and Schwinger-Keldysh techniques; the Einstein equation is therefore upgraded to a so-called Einstein-Langevin equation. We consider in some detail the conformal fluctuations of flat spacetime and the fluctuations of the scale factor in a simple cosmological model introduced by Hartle, which consists of a spatially flat isotropic cosmology driven by radiation and dust. [S0556-2821(97)04716-4]
\end{abstract}

PACS number(s): 04.62.+v, 05.40.+j, 98.80.Cq

\section{INTRODUCTION}

When a model of the early Universe is developed, it becomes necessary to take into account the quantum nature of matter fields, even if for a wide range of energies, leading up to Planck's, the geometry of the Universe itself may be described in classical terms. The simplest and most studied way to couple quantum matter to a classical gravitational field is through the expectation value of the energymomentum tensor of the quantum fields $[1,2]$; however, the quantum fields must necessarily undergo fluctuations $[3,4]$, and it has been recently shown that these fluctuations are by no means negligible in many situations of interest $[5,6]$.

To a certain extent, quantum fluctuations may be introduced in a classical model as uncertainty in the initial conditions. However, fluctuations play a subtler role when the semiclassical evolution, as is in fact the rule, is dissipative $[7,8]$. In this case, the semiclassical gravitational field interacts in a nontrivial way with the environment provided by the quantum matter degrees of freedom [9], leading, e.g., to anisotropy damping and loss of information regarding initial conditions [10-13]. The environment reacts back on the system in a way which is demanded by consistency of the underlying quantum theory. Under equilibrium conditions in static spacetimes, the relationship between damping and back reaction becomes rather simple, and it is embodied in the so-called fluctuation-dissipation theorems [14]. Similarly, if more complex, connections between damping and back reaction hold in nonequilibrium situations, then as a rule, the back reaction acquires a stochastic character [15].

In the particular case of semiclassical cosmology, we can be more precise about what is going on. Except in some very special cases, such as conformally flat universes coupled to conformal matter, or purely adiabatic evolution, the cosmo- logical evolution leads to particle creation [16,17]. The phase relationships between the created particles are quickly lost; even very weak interactions may accomplish this on very short time scales [18]. Under these conditions, particle creation is a dissipative process. The back reaction of the matter fields will then (loosely speaking) have a time reversible component associated to vacuum polarization phenomena (such as trace anomaly) and a time oriented component related to particle creation. The crucial point is that there is also a third, stochastic component, induced by the quantum fluctuations of the matter fields. To keep the average effect while disregarding these fluctuations leads, as a rule, to an inconsistent theory.

In recent years Langevin-type equations have been proposed to describe the fluctuating back reaction of the matter fields on the classical gravitational field, thus restoring the consistency of the semiclassical Einstein equations. The new semiclassical Einstein-Langevin equations predict classical stochastic fluctuations in the gravitational field $[8,15,19,20]$. The derivation of these improved semiclassical equations is based, usually, in the closed time path (CTP) effective action method and exploits the close connection that this effective action has with the influence functional introduced by Feynman and Vernon [21]. We should mention that a similar approach leading to Langevin type equations has been used in recent years to study the generation of primordial fluctuations in inflationary cosmologies [22-25] (Morikawa has independently arrived to similar conclusions regarding this subject [26]), as well as the more general problem of stochasticity in effective field theory [27].

In this paper we study the stochastic fluctuations arising from coupling geometry to massless nonconformal fields in two cases of interest. One of them is the study of the conformal fluctuations of flat spacetime. The second case is a 
spatially flat Friedmann-Robertson-Walker (FRW) spacetime with a classical source made of both radiation and dust. This model is simple enough that the fluctuations can be studied in some detail, yet it has significant features of a realistic cosmology; it was proposed some time ago by Hartle [28], within the usual (Rosenfeld's) approach, to study the backreaction effects to the creation of massless nonconformal particles in isotropic cosmologies. We shall not develop a full analysis, but rather focus on the new features brought by the explicit introduction of stochastic terms in the Einstein equations.

The Hartle model has two perfect fluids, one of them with the equation of state of radiation and the other representing baryonic matter with the equation of state of dust. A dimensionless parameter $\xi$ measuring the relative amounts of baryons and radiation, which is constant in classical periods, is assumed to be always constant and with a value corresponding to the present universe (which is very small, $\sim 10^{-27}$ ). A massless nonconformal quantum field is coupled to this system. The presence of the small portion of baryonic matter is essential to ensure particle creation, since in a radiative FRW universe the scalar curvature vanishes and the field cannot couple to the curvature. A perturbative expansion in terms of the parameter $\xi$ is then seen to be equivalent to an expansion in a parameter that measures the deviation from the conformal coupling. Since the two degrees of freedom of the graviton field in a FRW background behave as massless minimally coupled fields [29] Hartle's model provides a good testing ground for the study of the back reaction due to the creation of gravitons in cosmology.

The model describing the conformal fluctuations of flat spacetime due to massless nonconformal fields can be easily obtained as a limit of Hartle's model. One just needs to impose that there is no classical matter source and that the cosmological scale factor is a small perturbation of unity. This model, which is considerably simpler than the cosmological model, may be of interest in connection with the semiclassical stability of flat spacetime [30] (see also Ref. [31]) under conformal fluctuations.

We should stress that one of the main differences between the cosmological back-reaction problem considered in this paper and other cosmological back-reaction problems that have been considered in the literature is that the latter usually deal with conformal fields in isotropic cosmologies $[16,32,10]$, or with conformal fields in cosmologies with small anisotropies [33,34,17,35,11,12] or in cosmologies with small inhomogeneities [36,37]. In the two last cases particle creation takes place because the anisotropies or inhomogeneities break the conformal invariance of the fields.

Cosmological models where the back-reaction equations have been solved explicitly involve, usually, conformal fields in FRW cosmologies [38,39]. In such models there is no particle creation and all quantum effects are a consequence of the fact that the vacuum expectation value of the stressenergy tensor of the quantum field, subject to the trace anomaly, does not vanish. Generally the stress-energy tensor is also subject to a two parameter ambiguity connected to the addition of two conserved terms quadratic in the spacetime curvature $[2,40]$. Such ambiguity cannot be resolved within the semiclassical theory and depending on these parameters one finds very different behaviors for the cosmological scale factor. Different behaviors are also found as a consequence of the higher than two time derivatives of the scale factor that appear in the stress-energy tensor, this is a typical backreaction phenomenon and the origin of runaway solutions. In this connection several authors have argued $[41,31]$ that solutions to the semiclassical back-reaction equations that deviate nonperturbatively from the classical solutions should be discarded.

When particle creation takes place, the back-reaction equations have been solved only numerically for the case of massive conformally coupled fields in FRW cosmologies [42]. In this case it is the mass that breaks the conformal symmetry of the problem.

Here we are interested in the quantum effects due to the creation of massless nonconformal particles. Our dynamical back-reaction equations for the cosmological scale factor generalize that of Ref. [28] in that a new stochastic source is included. Because we start with a CTP functional formalism our equations are real and causal and thus they admit an initial value formulation. Thus when our equations are averaged with respect to the stochastic source they should not be directly compared with the equations in Ref. [28], which were obtained using an in-out functional formalism. However, the analysis carried out by Hartle on the behavior of the cosmological scale factor at large and very small cosmological times is still valid. For this reason we concentrate here mainly in the stochastic effects which are new.

The plan of the paper is the following. In Sec. II the actions for the massless nonconformal scalar field, the gravitational field, and the classical matter sources, which define Hartle's model, are described. In Sec. III we give the stochastic back-reaction equations for the cosmological scale factor. The derivation of these equations is based in the CTP effective action describing the interaction of the classical scale factor with the quantum field, and the relation of this effective action with the influence functional of Feynman and Vernon [21]. In Sec. IV we consider the particular case of the conformal perturbations of flat spacetime. In Sec. V we consider the fluctuation in the cosmological scale factor in Hartle's model and Sec. VI summarizes our conclusions.

\section{HARTLE'S MODEL}

In this section we describe a model [28] in which a massless nonconformal quantum scalar field is coupled to a spatially flat FRW cosmology with a classical source made of radiation and dust. Since we use a dimensional regularization technique some relevant terms in the classical action are given in $n$ arbitrary dimensions. The cosmological model is described by the $n$-dimensional spatially flat FRW metric

$$
d s^{2}=a^{2} \eta_{\mu \nu} d x^{\mu} d x^{\nu},
$$

where $a(\eta)$ is the cosmological scale factor, $\eta$ is the conformal time, and $\eta_{\mu \nu}=\operatorname{diag}(-1,1, \ldots, 1)$. The classical action for the massless nonconformally coupled scalar field $\Phi\left(x^{\mu}\right)$ is

$$
\begin{aligned}
S_{m}\left[g_{\mu \nu}, \Phi\right]= & -\frac{1}{2} \int d^{n} x \sqrt{-g}\left[g^{\mu \nu} \partial_{\mu} \Phi \partial_{\nu} \Phi\right. \\
& \left.+\left(\xi_{c}+\nu\right) R \Phi^{2}\right],
\end{aligned}
$$


where $R$ is the Ricci curvature scalar,

$$
R=2(n-1)\left[\frac{\ddot{a}}{a^{3}}+\left(\frac{n-4}{2}\right) \frac{\dot{a}^{2}}{a^{4}}\right],
$$

$\xi_{c}+\nu$ is the parameter coupling the scalar field to the spacetime curvature, and $\xi_{c} \equiv(n-2) / 4(n-1)$ is the coupling parameter for a conformal field in $n$ dimensions. Since the metric (2.1) is conformally flat it is convenient to introduce a new rescaled matter field $\phi\left(x^{\mu}\right)$ by $\phi\left(x^{\mu}\right)$ $\equiv a^{(n-2) / 2}(\eta) \Phi\left(x^{\mu}\right)$, and the scalar field action simplifies as

$$
S_{m}[a, \phi]=\frac{1}{2} \int d^{n} x \phi\left(x^{\mu}\right)\left[\square-\nu a^{2} R\right] \phi\left(x^{\mu}\right),
$$

where now $\square \equiv \eta^{\mu \nu} \partial_{\mu} \partial_{\nu}$ is the flat d'Alambertian operator. It is clear from this equation that if $\nu \neq 0$ the conformally flat symmetry is broken by the coupling of the scalar field.

The action for the gravitational field, the Einstein-Hilbert action $S_{g}^{\mathrm{EH}}$, needs to be corrected with a counterterm to cancel the divergencies that will come from the effective action. In our case it suffices to add a term quadratic in the Ricci scalar. Note that terms quadratic in $R_{\mu \nu}$ or the Weyl tensor could also be added but since these terms are not necessary for the renormalization we simply assume that their coefficients vanish. The divergent (in $n=4$ ) counterterm is

$$
S_{g}^{\mathrm{div}}\left[a ; \mu_{c}\right]=\frac{\nu^{2} \mu_{c}^{n-4}}{32 \pi^{2}(n-4)} \int d^{n} x \sqrt{-g} R^{2},
$$

where $\mu_{c}$ is an arbitrary mass scale needed to give the correct dimensions to this action term.

Since the only dynamical variable of the gravitational field is the cosmological scale factor $a(\eta)$ the gravitational action reduces in our case, when expanded in $(n-4)$, to

$$
\begin{aligned}
& S_{g}^{\mathrm{EH}}[a]=\frac{6 \mathcal{V}}{\ell_{P}^{2}} \int d \eta a \ddot{a}, \\
& S_{g}^{\mathrm{div}}\left[a ; \mu_{c}\right]=\frac{\nu^{2} \mathcal{V}}{32 \pi^{2}}\left[\frac{36}{n-4} \int d \eta\left(\frac{\ddot{a}}{a}\right)^{2}+36 \ln \mu_{c} \int d \eta\left(\frac{\ddot{a}}{a}\right)^{2}\right. \\
& +12 \int d \eta\left\{3\left(\frac{\ddot{a}}{a}\right)^{2} \ln a+\left(\frac{\ddot{a}}{a}\right)\left[3\left(\frac{\dot{a}}{a}\right)^{2}\right.\right. \\
& \left.\left.\left.+2\left(\frac{\ddot{a}}{a}\right)\right]\right\}\right]+O(n-4)
\end{aligned}
$$

where $\ell_{P}^{2}=16 \pi G_{N}\left(G_{N}\right.$ is the Newtonian gravitational constant) and $\mathcal{V}$ is the volume integral $\mathcal{V} \equiv \int d^{3} \mathbf{x}$.

To the above scalar and gravitational actions we need to add the action for the classical matter sources $S_{m}^{\mathrm{cl}}$, which are a radiative and dust perfect fluids. This action is given by

$$
S_{m}^{\mathrm{cl}}[a]=-\mathcal{V} \int d \eta \tilde{\rho}_{b} a,
$$

where $\tilde{\rho}_{b}$ is a constant parameter related to the baryon energy density. It is connected to a similar parameter $\tilde{\rho}_{r}$ for the energy density of the radiation, through

$$
\xi=\frac{\ell_{P} \widetilde{\rho}_{b}}{\widetilde{\rho}_{r}^{3 / 4}},
$$

which is of order $\xi \sim 10^{-27}$ for the present universe. This parameter $\xi$ measures the baryon to photon ratio.

The action (2.6) is justified because when it is varied with respect to the scale factor $a(\eta)$ it reproduces the trace of the stress-energy tensor for a radiative perfect fluid with equation of state $p_{r}=\rho_{r} / 3\left(p_{r}\right.$ is the pressure and $\rho_{r}$ is the energy density of the radiation), and of a dustlike baryon fluid of pressure $p_{b}=0$ and energy density $\rho_{b}$. When the dynamics of the scale factor is driven by the classical source only one finds that $\rho_{b}={\widetilde{\rho_{b}}}_{b} a^{-3}$ and $\rho_{r}={\widetilde{\rho_{r}}}_{r} a^{-4}$, then the relative amounts of baryons and radiation as defined by $\ell_{p} \rho_{b} / \rho_{r}^{3 / 4}$ becomes constant and given by Eq. (2.7). In fact, when the classical action (2.6) is considered as the only dynamical source of gravity from the Einstein-Hilbert action (2.4) one gets $6 \ddot{a}=\left(\ell_{P}^{2} / 2\right) \tilde{\rho}_{b}$ or equivalently $R=-\left(\ell_{P}^{2} / 2\right) T^{\mathrm{cl}}$, where $T^{\mathrm{cl}}=-\rho_{b}$ is the trace of the stress-energy tensor of a perfect fluid of dust (baryons) plus radiation.

We note that when baryons are not present, i.e., when $\xi=0$, the scalar curvature vanishes $(R=0)$, and from the action (2.2) for the scalar field $\phi\left(x^{\mu}\right)$ it is clear that this field behaves as a free field in flat spacetime. Therefore with the usual Minkowskian definition of a vacuum state [which corresponds to the conformal vacuum for $\left.\Phi\left(x^{\mu}\right)\right]$ no particles can be created by the expansion of the Universe. Backreaction effects are still possible due to the vacuum expectation value of the stress-energy tensor of the scalar field, which in this case depends on the two free parameters mentioned in the Introduction. One of the parameters is associated to an action proportional to $R^{2}$ and the other to an action proportional to $R_{\mu \nu} R^{\mu \nu}$. The quadratic terms of the first parameter vanish when $R=0$, but the quadratic terms of the second parameter are generally different from zero even when the scalar curvature vanishes. Thus all quantum effects in this case depend crucially on the second parameter being different from zero. We assume that such parameter is zero, thus $R=0$ is a consistent solution to the semiclassical backreaction problem. Since our interest is in the back reaction due to particle creation we will take $\xi$ as a small nonzero parameter, and we will consider the $\nu a^{2} R$ term in Eq. (2.2) as a perturbative term which gives a measure of the deviation from the radiative case $R=0$. In practice we will compute the quantum corrections as perturbations in powers of the parameter $\nu$, and this should be consistent with an expansion in $\xi$.

\section{STOCHASTIC BACK-REACTION EQUATIONS}

In this section we compute the stochastic back-reaction equations for the cosmological scale factor $a(\eta)$ from the CTP effective action to one-loop order for the quantum field $\Phi\left(x^{\mu}\right)$ in the FRW spacetime (2.1). This action is evaluated using a perturbative expansion in powers of the parameter $\nu$. The CTP effective action was introduced by Schwinger 
[43-45]; for its application in a curved background see Refs. $[12,7,46,37]$. The idea is to start with a generating functional from which one obtains expectation values, instead of the matrix elements one obtains using the generating functional of the ordinary in-out effective action [47]. The CTP effective action is the Legendre transform of such a generating functional. The price one pays for this is that one needs to double the number of fields: the $(+)$ and $(-)$ fields below. For the details of this paper we follow closely Ref. [37]; see also Ref. [48].

In our case we have two fields $a(\eta)$ and $\phi\left(x^{\mu}\right)$ whose free actions are given by $S_{g}\left[a ; \mu_{c}\right]+S_{m}^{\mathrm{cl}}[a]$ from Eqs. (2.4)(2.6), and by $S_{m}^{\text {free }}[\phi]=\frac{1}{2} \int d^{n} x \phi \square \phi$ from Eq. (2.2), and an interaction action given by $S_{\text {int }}[a, \phi]=-(\nu / 2) \int d^{n} x a^{2} R \phi^{2}$. The field $a$ is classical, thus we only need to go up to one loop order for the field $\phi$, which corresponds to the firstorder expansion of the generating functional in powers of $\hbar$. We have

$$
\begin{aligned}
\Gamma_{\mathrm{CTP}}\left[a^{ \pm}, \bar{\phi}_{ \pm}\right] \simeq & S_{g}\left[a^{+} ; \mu_{c}\right]-S_{g}\left[a^{-} ; \mu_{c}\right]+S_{m}^{\mathrm{cl}}\left[a^{+}\right] \\
& -S_{m}^{\mathrm{cl}}\left[a^{-}\right]+S_{m}\left[a^{+}, \bar{\phi}_{+}\right]-S_{m}\left[a^{-}, \bar{\phi}_{-}\right] \\
& -\frac{i}{2} \operatorname{Tr}(\ln G),
\end{aligned}
$$

where the fields $\bar{\phi}_{ \pm}$correspond to the expectation values of $\phi_{ \pm}$, and $G$, which is a matrix operator with $2 \times 2$ components, is the inverse of the classical kinetic operator $A=\operatorname{diag}\left\{\square-\nu\left(a^{+}\right)^{2} R^{+},-\left[\square-\nu\left(a^{-}\right)^{2} R^{-}\right]\right\}, \quad$ obtained from Eq. (2.2), with CTP boundary conditions (see [12]). Since we are only interested in the effective action for the scale factor $a(\eta)$, we set $\bar{\phi}_{ \pm}=0$ and expand the functional determinant in powers of the coupling constant; finally [48], the renormalized CTP effective action reads

$$
\Gamma_{\mathrm{CTP}}\left[a^{ \pm}\right] \simeq S_{g, m}^{R}\left[a^{+}\right]-S_{g, m}^{R}\left[a^{-}\right]+S_{\mathrm{IF}}^{R}\left[a^{ \pm}\right],
$$

where

$$
S_{g, m}^{R}[a]=\mathcal{V} \int d \eta\left[-\frac{6}{\ell_{p}^{2}} \dot{a}^{2}+\frac{9 \nu^{2}}{8 \pi^{2}}\left(\frac{\ddot{a}}{a}\right)^{2} \ln a-\tilde{\rho}_{b} a\right]
$$

and

$$
\begin{aligned}
S_{\mathrm{IF}}^{R}\left[a^{ \pm}\right]= & \frac{9 \nu^{2}}{8 \pi^{2}} \int d \eta d \eta^{\prime} \Delta\left(\frac{\ddot{a}}{a}\right)(\eta) \mathrm{H}\left(\eta-\eta^{\prime} ; \bar{\mu}\right)\left\{\left(\frac{\ddot{a}}{a}\right)\left(\eta^{\prime}\right)\right\} \\
& +36 \nu^{2} i \int_{-\infty}^{\infty} d \eta \int_{-\infty}^{\eta} d \eta^{\prime} \Delta\left(\frac{\ddot{a}}{a}\right) \\
& \times(\eta) \mathrm{N}\left(\eta-\eta^{\prime}\right) \Delta\left(\frac{\ddot{a}}{a}\right)\left(\eta^{\prime}\right),
\end{aligned}
$$

where we use the notation $\Delta f(\eta) \equiv f_{+}(\eta)-f_{-}(\eta)$, $\{f(\eta)\} \equiv f_{+}(\eta)+f_{-}(\eta)$, for an arbitrary function $f(\eta)$. The kernels $\mathrm{H}\left(\eta-\eta^{\prime} ; \bar{\mu}\right)$ and $\mathrm{N}\left(\eta-\eta^{\prime}\right)$ are

$$
\begin{gathered}
\mathrm{H}\left(\eta-\eta^{\prime} ; \bar{\mu}\right)=-\frac{\mathcal{V}}{2} \int \frac{d p^{0}}{2 \pi} e^{-i p^{0}\left(\eta-\eta^{\prime}\right)} \ln \left[-\left(\frac{p^{o}+i \varepsilon}{\bar{\mu}}\right)^{2}\right] \\
\mathrm{N}\left(\eta-\eta^{\prime}\right)=\frac{\mathcal{V}}{32 \pi} \delta\left(\eta-\eta^{\prime}\right)
\end{gathered}
$$

Note that the parameter $\mu_{c}$ has been absorved into the new parameter $\bar{\mu}$.

The subscript IF in $S_{\mathrm{IF}}^{R}\left[a^{ \pm}\right]$is used to indicate that this part of the CTP effective action is the influence functional action as defined by Feynman and Vernon [21]. That is, it gives the effect of the environment, the quantum field, on the system, the cosmological scale factor, which is the field of interest here. The identification between a part of the CTP effective action and the influence functional was suggested in Ref. [8].

\section{A. Improved effective action}

The connection between the CTP effective action in the semiclassical context and the influence functional action introduced by Feynman and Vernon to describe the interaction between a system and an environment just mentioned gives an interesting new light on the semiclassical back-reaction problem.

Let us now turn to the dynamical equations for the scalar field $a(\eta)$. In principle these equations can be derived from the CTP effective action as $\delta \Gamma_{\mathrm{CTP}} /\left.\delta a^{+}\right|_{a^{ \pm}=a}=0$. A problem might arise from the fact that such effective action has an imaginary part, since $S_{\mathrm{IF}}^{R}$ is complex, see Eq. (3.4). It should be clear, however, that the imaginary part of $S_{\mathrm{IF}}^{R}$ will not contribute to the field equation derived in that form because $\operatorname{Im}\left(S_{\mathrm{IF}}^{R}\left[a^{ \pm}\right]\right)$is quadratic in the difference of the $(+)$and ( - ) fields and one finally takes $a^{+}=a^{-}=a$.

However, from the point of view of the system environment, relation familiar in quantum statistical mechanics, the imaginary part of the influence action is related to the noise suffered by the system from the environment fluctuations [21]. This suggests an improvement to the semiclassical back-reaction equations that takes into account such fluctuations. This may be achieved if we define the influence functional [21]

$$
\mathcal{F}_{\mathrm{IF}}\left[a^{ \pm}\right]=e^{i S_{\mathrm{IF}}^{R}\left[a^{ \pm}\right]},
$$

and note that it may be written formally as

$$
\begin{aligned}
\mathcal{F}_{\mathrm{IF}}\left[a^{ \pm}\right]= & \int \mathcal{D} j \mathcal{P}[j] \exp \left\{i \left[\operatorname{Re}\left(S_{\mathrm{IF}}^{R}\left[a^{ \pm}\right]\right)\right.\right. \\
& \left.\left.+6 \nu \int d \eta j(\eta) \Delta\left(\frac{\ddot{a}}{a}\right)(\eta)\right]\right\},
\end{aligned}
$$

with

$$
\mathcal{P}[j]=\frac{e^{-(1 / 2) \int d \eta d \eta^{\prime} j(\eta)\left[\mathrm{N}\left(\eta-\eta^{\prime}\right)\right]^{-1} j\left(\eta^{\prime}\right)}}{\int \mathcal{D} j e^{-(1 / 2) \int d \eta d \eta^{\prime} j(\eta)\left[\mathrm{N}\left(\eta-\eta^{\prime}\right)\right]^{-1} j\left(\eta^{\prime}\right)}} .
$$


That is, performing the path integral in Eq. (3.7) with $\mathcal{P}[j]$ defined in Eq. (3.8) leads directly to Eq. (3.6). If we interpret $\mathcal{P}[j]$ as a Gaussian probability distribution, the action in Eq. (3.7) may be seen formally as the action for a field $a(\eta)$ which is coupled to an external stochastic source $j(\eta)$.

Thus, the influence functional (3.6) can be seen as the mean value with respect to the stochastic field $j(\eta)$ of an influence functional for an improved effective action $S_{\text {eff }}$ defined by

$$
\begin{aligned}
S_{\mathrm{eff}}\left[a^{ \pm} ; j\right]= & S_{g, m}^{R}\left[a^{+}\right]-S_{g, m}^{R}\left[a^{-}\right]+\operatorname{Re}\left(S_{\mathrm{IF}}^{R}\left[a^{ \pm}\right]\right) \\
& +6 \nu \int d \eta j(\eta) \Delta\left(\frac{\ddot{a}}{a}\right)(\eta) .
\end{aligned}
$$

This comes from Eq. (3.7) and the addition of the gravitational and classical matter terms (3.3). Now the field $j(\eta)$ will act as a stochastic source in the improved semiclassical equation when the functional derivation with respect to $a^{+}(\eta)$ is taken. This stochastic field is not dynamical, it is completely determined by the following relations, which may be derived from the characteristic functional, i.e., the functional Fourier transform of $\mathcal{P}[j]$ :

$$
\begin{gathered}
\langle j(\eta)\rangle=0, \\
\left\langle j(\eta) j\left(\eta^{\prime}\right)\right\rangle=\mathrm{N}\left(\eta-\eta^{\prime}\right) .
\end{gathered}
$$

Since the probability distribution is Gaussian the noise kernel is the two point correlation function of the stochastic field. In our case as one can see from Eq. (3.5) the noise is white.

It is convenient for comparison with previous work $[28,39]$, to write the effective action (3.9) in terms of dimensionless quantities such as $\chi, b(\chi)$, and $\zeta(\chi)$ for the conformal time $\eta$, cosmological scale factor $a(\eta)$, and stochastic source $j(\eta)$, respectively. These are defined by

$$
\chi=\frac{\widetilde{\rho}_{r}^{1 / 4}}{6^{1 / 2}} \eta, \quad b=\frac{a}{\ell_{P} \widetilde{\rho}_{r}^{1 / 4}}, \quad \zeta=\frac{\widetilde{\rho}_{r}^{1 / 4}}{6^{1 / 2}} j .
$$

We also introduce dimensionless frequencies $\omega$, renormalization parameter $\mu$, and volume $V$ instead of $p^{0}, \bar{\mu}$, and $\mathcal{V}$, respectively, as

$$
\omega=\frac{6^{1 / 2}}{\widetilde{\rho}_{r}^{1 / 4}} p^{0}, \quad \mu=6^{1 / 2} \ell_{P} \bar{\mu}, \quad \mathcal{V}=\frac{\widetilde{\rho}_{r}^{3 / 4}}{6^{1 / 2}} \mathcal{V} .
$$

Then the improved effective action (3.9) for the dimensionless scale field $b(\chi)$ becomes

$$
\begin{aligned}
S_{\mathrm{eff}}\left[b^{ \pm} ; \zeta\right] \equiv & \mathcal{V} 6^{1 / 2} \widetilde{\rho}_{r}^{3 / 4} \int d \chi\left\{-\Delta\left(\dot{b}^{2}+\xi b\right)(\chi)\right. \\
& +\frac{\nu^{2}}{32 \pi^{2}}\left[\Delta\left(\left(\frac{\dot{b}}{b}\right)^{2} \ln b\right)(\chi)-\Delta\left(\frac{\ddot{b}}{b}\right)\right. \\
& \left.\left.\times(\chi) \kappa\left[\chi ;\left\{\frac{\ddot{b}}{b}\right\}\right]\right]+\frac{\nu}{V} \zeta(\chi) \Delta\left(\frac{\ddot{b}}{b}\right)(\chi)\right\} .
\end{aligned}
$$

The stochastic source $\zeta(\chi)$ is defined, after Eq. (3.10), by $\langle\zeta(\chi)\rangle=0$ and

$$
\left\langle\zeta(\chi) \zeta\left(\chi^{\prime}\right)\right\rangle=\frac{V}{192 \pi} \delta\left(\chi-\chi^{\prime}\right) .
$$

The nonlocal operator $\kappa$ is defined by its action on a test function $f(\chi)$ by

$$
\kappa[\chi ; f(\chi)] \equiv \int_{-\infty}^{\infty} \frac{d \omega}{2 \pi} e^{-i \omega \chi} h(\omega) f(\omega)
$$

where $f(\omega)$ is the Fourier transform of the test function, defined by $f(\omega)=\int_{-\infty}^{\infty} d \chi e^{i \omega \chi} f(\chi)$, and

$$
h(\omega) \equiv \frac{1}{2} \ln \left[-\left(\frac{\omega+i \varepsilon}{\mu}\right)^{2}\right] .
$$

This kernel is real and causal, as can be seen from its analytical structure. Note that this is in fact the main difference between the CTP approach and Hartle's approach.

\section{B. Stochastic back-reaction equations}

The dynamical equations for the cosmological scale factor in its dimensionless form, $b(\chi)$, may be obtained by functional derivation of the effective action (3.13) as $(\delta /$ $\left.\delta b^{+}\right)\left.\left(S_{\text {eff }}\left[b^{ \pm} ; j\right]\right)\right|_{b^{ \pm}=b} \equiv 0$. After functional derivation and the identification of the $(+)$ and $(-)$ fields, the equation acquire an interesting form if we multiply it by $\dot{b}$ :

$$
\begin{gathered}
\frac{d}{d \chi}\left\{\dot{b}^{2}-\xi b-\frac{\nu^{2}}{32 \pi^{2}}\left(\frac{\ddot{b}}{b}\right)^{2} \ln b+\dot{b} \frac{d}{d \chi}\left[\frac { \nu ^ { 2 } } { 1 6 \pi ^ { 2 } } \left[\frac{\ddot{b}}{b^{2}} \ln b\right.\right.\right. \\
\left.\left.\left.-\frac{1}{b} \kappa\left[\chi ; \frac{\ddot{b}}{b}\right]\right]+\frac{\nu}{V} \frac{\zeta(\chi)}{b}\right]\right\} \\
=\frac{\ddot{b}}{b} \frac{d}{d \chi}\left[-\frac{\nu^{2}}{16 \pi^{2}} \kappa\left[\chi ; \frac{\ddot{b}}{b}\right]+\frac{\nu}{V} \zeta(\chi)\right] .
\end{gathered}
$$

The effects of the quantum field are those proportional to the coupling parameter $\nu$. The terms with $\ln b$ come from the renormalization of the stress-energy tensor of the quantum field, see Eq. (2.5), and there are nonlocal contributions in the terms with $\kappa[\chi ; \ddot{b} / b]$. The effects due to the creation of quantum particles, in particular the dissipation of the field $b(\chi)$ are included in the nonlocal term.

A point of interest is the role played by the parameter $\mu$ in Eq. (3.17).This parameter is related to the local part of 
$\kappa[\chi ; f(\chi)]$, thus a change in this parameter is equivalent to a change in the coefficients in some local terms in the stressenergy momentum tensor of the quantum field. This parameter in fact is related to the two parameter ambiguity in the definition of such stress-energy tensor already mentioned. In our case one of the parameters has been fixed by our renormalization procedure and the ambiguity remains in the other parameter, see Ref. [31] for more details.

When quantum effects are ignored, i.e., when we take $\nu=0$, the back-reaction equation becomes $2 b-\bar{\xi}=0$. This is the classical equation when only the two classical fluids are present; it admits the solution

$$
b=\chi+\frac{1}{4} \xi \chi^{2},
$$

with appropriate initial conditions. When baryonic matter is not present, i.e., $\xi=0$, the classical solution is $b=\chi$. If averaged with respect to $\zeta(\chi)$, Eq. (3.17) also admits $b=\chi$ as a solution, which is in agreement with Hartle's solution. As remarked in the Introduction this is expected as in such a case no particle creation takes place. Had we introduced local terms in the action of the type $R_{\mu \nu} R^{\mu \nu}$, this would not be necessarily the case.

\section{CONFORMAL FLUCTUATIONS IN FLAT SPACETIME}

The semiclassical back-reaction equation (3.17) was derived for a nonconformal quantum field in a universe filled with classical radiation and baryonic matter, using perturbation theory around a conformal vacuum state. The classical contributions are in the first two terms of the equation, i.e., $\dot{b}^{2}-\xi b$. These two terms are obtained from the classical stress-energy tensor and different classical sources will lead to different terms. Thus by changing these first two terms in Eq. (3.17), the equation may be used in different situations of interest. Besides the case of cosmological interest described in the previous section, a case of obvious interest is that of the conformal fluctuations of flat spacetime.

To obtain the equations for the fluctuations around flat spacetime, one simple takes $\xi=0$ (no baryons) and notes that $b=1$ (or $a=1$ ) is the conformal factor for flat spacetime. Thus if we take

$$
b(\chi)=1+g(\chi)
$$

in Eq. (3.17) with $\xi=0$ and consider the linear order in $g(\chi)$ we find an equation for the conformal fluctuations of flat spacetime, interacting with a massless nonconformally coupled scalar field. Equation (3.17) in this linear approximation simplifies considerably, it can be integrated twice and becomes

$$
g-\frac{\nu^{2}}{32 \pi^{2}} \kappa[\chi ; \ddot{g}]+\frac{\nu}{2 V} \zeta(\chi)=A_{0} \chi+B_{0},
$$

where $A_{0}$ and $B_{0}$ are integration constants, $B_{0}$ gives just a global rescaling of the conformal factor, and $A_{0}$ gives a linear expansion of the scale factor. This linear term is pure gauge, it represents an infinitesimal coordinate change of $t \rightarrow t+\left(A_{0} / 2\right) t^{2}$, where $t$ is the Minkowski time. We can take
$A_{0}=B_{0}=0$ as our background spacetime or, alternatively, define a new perturbation of the scale factor $G(\chi) \equiv g(\chi)-A_{0} \chi-B_{0}$, whose equation is

$$
G(\chi)-\frac{\nu^{2}}{32 \pi^{2}} \kappa[\chi ; \ddot{G}]+\frac{\nu}{2 V} \zeta(\chi)=0 .
$$

The classical solution corresponds now to $G(\chi)=0$ (no perturbation). The quantum effects here reduce to the two terms which depend on $\nu$ in Eq. (4.3). The first, of order $\nu^{2}$, is a nonlocal term which is linear in $\ddot{G}(\chi)$, and the second is the external stochastic source $\zeta(\chi)$. The first includes the effects of particle creation, and the second drives the classical stochastic fluctuations of the conformal factor. To solve this equation we may take its Fourier transform,

$$
G(\omega)=-\frac{\nu}{2 V}\left[\frac{\zeta(\omega)}{1+\left(\nu^{2} / 32 \pi^{2}\right) \omega^{2} h(\omega)}\right],
$$

where $h(\omega)$ is given in Eq. (3.16). From Eq. (3.14) we know that

$$
\left\langle\zeta(\omega) \zeta\left(\omega^{\prime}\right)\right\rangle=\frac{V}{96} \delta\left(\omega+\omega^{\prime}\right),
$$

and so

$$
\left\langle G(\omega) G\left(\omega^{\prime}\right)\right\rangle=\frac{\nu^{2}}{384 V}\left|1+\frac{\nu^{2} \omega^{2}}{32 \pi^{2}} h(\omega)\right|^{-2} \delta\left(\omega+\omega^{\prime}\right),
$$

which leads to the real time correlation

$$
\left\langle G(\chi) G\left(\chi^{\prime}\right)\right\rangle=\frac{\nu^{2}}{768 \pi V} \int \frac{d \omega}{2 \pi\left|1+\left(\nu^{2} \omega^{2} / 32 \pi^{2}\right) h(\omega)\right|^{-i \omega\left(\chi^{-} \chi^{\prime}\right)}} .
$$

As $\nu \rightarrow 0$, the conformal stochastic fluctuations become white noise, as it could be expected. Indeed, we could Taylor expand inside the integration sign to develop the correlation function as a formal power series in $\nu$. An important problem, however, of this approximation is that the fluctuation effect $[$ in $\zeta(\chi)]$ and the dissipation effect (in $\kappa[\chi ; \ddot{G}]$ ) are treated at different orders of approximation and this means that the fluctuation-dissipation relation cannot be fully appreciated. In fact, one would expect that as in the Brownian motion the scale factor driven by the stochastic term will loose energy by dissipation due to particle creation and that finally a sort of equilibrium between the two effects will be reached. Here the stochastic effect dominates the behavior of $b(\chi)$.

We may estimate the correlation time at finite $\nu$ by rescaling $\omega=(8 \pi / \nu) s$; we find

$\left\langle G(\chi) G\left(\chi^{\prime}\right)\right\rangle=\frac{\nu}{96 V} \int_{-\infty}^{\infty} \frac{d s}{2 \pi} \frac{e^{-i 8 \pi \nu^{-1} s\left(\chi-\chi^{\prime}\right)}}{\left[1+s^{2} \ln (8 \pi s / \mu \nu)^{2}\right]^{2}+\pi^{2} s^{4}}$.

The integral is controlled by the small $s$ range. Recalling that the function $x \ln (x / e)$ has a minimum at $x=1$, we expand 


$$
1+x \ln \left(\frac{\lambda x}{e}\right) \sim 1-\frac{1}{\lambda}+\frac{\lambda}{2}\left(x-\frac{1}{\lambda}\right)^{2} \sim 1+\frac{\lambda}{2}\left(x-\frac{1}{\lambda}\right)^{2},
$$

where $\lambda=e(8 \pi / \mu \nu)^{2}$, and we have used that $\lambda$ is a large number. In this approximation

$$
\begin{aligned}
{\left[1+s^{2} \ln \left(\frac{8 \pi s}{\mu \nu}\right)^{2}\right]^{2}+\pi^{2} s^{4} } & \sim 1+\lambda\left(s^{2}-\frac{1}{\lambda}\right)^{2}+\pi^{2} s^{4} \\
& \sim\left[\lambda+\pi^{2}\right] s^{4}-2 s^{2}+1
\end{aligned}
$$

The denominator has poles at

$$
s^{2}=\frac{1}{\lambda+\pi^{2}}\left[ \pm i \sqrt{\lambda+\pi^{2}-1}+1\right]
$$

which converge towards

$$
s \sim\left[\lambda+\pi^{2}\right]^{-1 / 4} \exp (1+2 k) \frac{i \pi}{4}
$$

( $k=0,1,2,3)$ as $\lambda \rightarrow \infty$. Choosing the contour of integration, we find

$$
\begin{aligned}
\left\langle G(\chi) G\left(\chi^{\prime}\right)\right\rangle \sim & \frac{\nu^{2}}{768 \pi V} \frac{\Theta}{\sqrt{2}} \cos \left[\Theta\left|\chi-\chi^{\prime}\right|-\frac{\pi}{4}\right] \\
& \times \exp \left[-\Theta\left|\chi-\chi^{\prime}\right|\right],
\end{aligned}
$$

where

$$
\Theta=\frac{4 \sqrt{2} \pi}{\nu\left[\lambda+\pi^{2}\right]^{1 / 4}}
$$

We see that at finite $\nu$ the correlation decays exponentially with a correlation time $\Theta^{-1} \sim \sqrt{\nu}$.

\section{FLUCTUATIONS IN A UNIVERSE WITH MATTER AND RADIATION}

We turn now to our main problem, which is the analysis of the fluctuations of the cosmological scale factor in Hartle's model, as described by Eq. (3.17).

The analytic solution of the stochastic integrodifferential equation (3.17) is not possible, but since $\xi$, the parameter that gives the baryon to photon ratio, is assumed to be very small, it makes sense to find solutions linearized around a radiative universe. Thus we follow Hartle [28] and look for solutions of the type

$$
b(\chi)=\chi+\xi g(\chi) .
$$

Substituting this into Eq. (3.17), taking only terms linear in $\xi$, the equation can be integrated twice and becomes

$$
g+\frac{\nu^{2}}{32 \pi^{2} \chi}\left(\frac{\ddot{g}}{\chi} \ln \chi-\kappa\left[\chi ; \frac{\ddot{g}}{\chi}\right]\right)+\frac{\nu}{2 V \xi} \frac{\zeta}{\chi}=\frac{\chi^{2}}{4}+A_{0} \chi+B_{0},
$$

where $A_{0}$ and $B_{0}$ are integration constants which may be taken as $A_{0}=B_{0}=0$, without loss of generality. The term $\chi^{2} / 4$ gives the expansion corresponding to a matter domi- nated universe and comes from the second term in Eq. (3.17). The terms with $\nu$ are of quantum origin. Equation (5.2) is the dynamical equation for the perturbation $g(\chi)$ of the scale factor around a radiative classical solution.

This equation can now be directly compared with Eq. (3.9) in Ref. [28]. In our case we have the external stochastic source $\zeta$ which accounts for the fluctuations of the quantum stress-energy tensor and was not considered previously, but if we take the mean value with respect of $\zeta$, the resulting equation is similar to Hartle's equation. The main difference here, as we pointed out earlier, is that the equation is real and causal. But the basic structure of the equation is similar and the conclusions that Hartle's draws in his analysis apply also here.

In our case, however, we want to concentrate in the stochastic terms (which are new) and the dissipative terms, and how they contribute to the dynamics of $g(\chi)$. It is convenient to consider separately the behavior of fluctuations far away from and close to the cosmological singularity.

\section{A. Fluctuations far from the singularity}

To study the behavior of the solutions to the EinsteinLangevin equation, we first decompose $g$ into the deterministic part $g_{d}$ and the stochastic component $g_{s}$. The behavior of the deterministic part follows Hartle's original analysis [28], leading to $g_{d} \sim \chi^{2} / 4+0\left(\ln \chi / \chi^{2}\right)$ for large $\chi$.

As for the stochastic part, it is convenient to adopt as dynamical variable $f=\ddot{g}_{s} / \chi$, rather than $g_{s}$ itself. The variable $f$ satisfies

$$
\begin{aligned}
\int{ }^{\chi} d \chi^{\prime} \chi \chi^{\prime}\left(\chi-\chi^{\prime}\right) f\left(\chi^{\prime}\right) \\
\quad+\frac{\nu^{2}}{32 \pi^{2}}\{f \ln \chi-\kappa[\chi ; f]\}=-\frac{\nu}{2 V \xi} \zeta .
\end{aligned}
$$

Instead of solving directly for $f$, it is convenient to look at the propagator

$$
G\left(\chi, \chi^{\prime}\right)=\frac{\delta f(\chi)}{\delta \zeta\left(\chi^{\prime}\right)}
$$

which obeys the equation

$$
\begin{gathered}
\int{ }^{\chi} d \chi^{\prime \prime} \chi \chi^{\prime \prime}\left(\chi-\chi^{\prime \prime}\right) G\left(\chi^{\prime \prime}, \chi^{\prime}\right)+\frac{\nu^{2}}{32 \pi^{2}}\left\{\ln \chi G\left(\chi, \chi^{\prime}\right)\right. \\
\left.-\kappa\left[\chi ; G\left(\chi, \chi^{\prime}\right)\right]\right\}=-\frac{\nu}{2 V \xi} \delta\left(\chi-\chi^{\prime}\right)
\end{gathered}
$$

The main formal difference between this case and the conformal fluctuations of flat space time lies in that this equation is not time translation invariant. However, as in the previous case we expect the propagator to decay exponentially on the difference $u=\chi-\chi^{\prime}$, with a weaker dependence on some "center-of-mass" variable, which we take for simplicity to be $X=\left(\chi+\chi^{\prime}\right) / 2$. Our problem is then to disentangle the fast variable $u$ from the slow one $X[49,50]$. Now observe that, since we expect $G(X, u)$ to be exponentially small unless $u \leqslant X$, we may approximate 


$$
\ln \chi G\left(\chi, \chi^{\prime}\right) \sim \ln X G(X, u) .
$$

On the other hand, let us write

$$
\kappa\left[\chi ; G\left(\chi, \chi^{\prime}\right)\right] \equiv \int d \chi^{\prime \prime} h\left(\chi^{-} \chi^{\prime \prime}\right) G\left(\chi^{\prime \prime}, \chi^{\prime}\right),
$$

and set $\chi^{\prime \prime}=\chi^{\prime}+v$ to get

$$
\kappa\left[\chi ; G\left(\chi, \chi^{\prime}\right)\right] \equiv \int d v h(u-v) G\left(X+\frac{v-u}{2}, v\right) .
$$

Assuming that $G$ depends weakly on $X$, we obtain

$$
\kappa\left[\chi ; G\left(\chi, \chi^{\prime}\right)\right] \sim \int d v h(u-v) G(X, v) .
$$

Handling the classical term in the same way,

$$
\int^{\chi} d \chi^{\prime \prime} \chi \chi^{\prime \prime}\left(\chi-\chi^{\prime \prime}\right) G\left(\chi^{\prime \prime}, \chi^{\prime}\right) \sim X^{2} \int^{u} d v(u-v) G(X, v),
$$

we obtain the translation invariant equation

$$
\begin{gathered}
X^{2} \int^{u} d v(u-v) G(X, v)+\frac{\nu^{2}}{32 \pi^{2}}\{\ln X G(X, u) \\
-\kappa[u ; G(X, u)]\}=-\frac{\nu}{2 V \xi} \delta(u),
\end{gathered}
$$

where $X$ plays the role of a parameter. The solution reads

$$
G(X, u)=\frac{\nu}{2 V \xi} \int \frac{d \omega}{2 \pi} e^{-i \omega u} G(X, \omega),
$$

where

$$
G(X, \omega)=\left\{\frac{X^{2}}{(\omega+i \varepsilon)^{2}}+\frac{\nu^{2}}{64 \pi^{2}} \ln \left[-\left(\frac{\omega+i \varepsilon}{\mu X}\right)^{2}\right]\right\}^{-1},
$$

remarkably similar to its flat space counterpart. The selfcorrelation is given in terms of the propagator as

$$
\left\langle f(\chi) f\left(\chi^{\prime}\right)\right\rangle=\frac{V}{192 \pi} \int d \chi^{\prime \prime} G\left(\chi, \chi^{\prime \prime}\right) G\left(\chi^{\prime}, \chi^{\prime \prime}\right),
$$

which, with the same degree of accuracy, becomes

$$
\left\langle f(\chi) f\left(\chi^{\prime}\right)\right\rangle=\frac{\nu^{2}}{768 \pi V \xi^{2}} \int \frac{d \omega}{2 \pi} e^{-i \omega\left(\chi-\chi^{\prime}\right)}|G(X, \omega)|^{2} .
$$

We can repeat for this self-correlation the same analysis than in the previous section, provided we replace $(\nu / X)$ for $\nu$ and $\mu X$ for $\mu$, to get

$$
\begin{aligned}
\left\langle g_{s}(\chi) g_{s}\left(\chi^{\prime}\right)\right\rangle \sim & \frac{\nu^{2}}{768 \pi V \xi^{2} X^{2}} \frac{\Theta_{c}}{\sqrt{2}} \cos \left[\Theta_{c}\left|\chi-\chi^{\prime}\right|-\frac{\pi}{4}\right] \\
& \times \exp \left[-\Theta_{c}\left|\chi-\chi^{\prime}\right|\right]
\end{aligned}
$$

where $\Theta_{c}=X \Theta$. As a result, the correlation conformal time $\Theta_{c}^{-1}$ scales as $\sqrt{\nu} / X$. Of course, the approximations we have made assume that this correlation conformal time is small compared to $X$ itself $\left(X \geqslant \nu^{1 / 4}\right)$. In the region of validity, we see that the effect of expansion is to shorten the correlation time.

\section{B. Fluctuations near the singularity}

To obtain the form of the solutions valid at early times, we reason as follows. When $\chi$ is small, the Fourier integral defining the nonlocal part of the Einstein-Langevin equation is dominated by large frequencies $\omega \sim \chi^{-1}$. But since $h(\omega)$ depends only logarithmically on the frequency, we can then set $h(\omega)=$ const $=h\left(\chi^{-1}\right)$, to obtain

$$
\kappa[\chi ; f(\chi)] \sim-\ln [\mu \chi] f(\chi) .
$$

The Einstein-Langevin equation then becomes

$$
\chi^{2} g+\frac{\nu^{2}}{16 \pi^{2}} \ln \left(\frac{\chi}{\chi_{0}}\right) \ddot{g}+\frac{\nu \chi}{2 V \xi} \zeta=\frac{\chi^{4}}{4},
$$

where $\chi_{0}=\mu^{-1 / 2}$. Writing $\chi=\chi_{0} e^{t^{2} / 2}$, we find

$$
\begin{gathered}
\frac{\nu^{2}}{32 \pi^{2}}\left[\frac{d^{2}}{d t^{2}}-\left(\frac{1+t^{2}}{t}\right) \frac{d}{d t}\right] g+\chi_{0}^{4} e^{2 t^{2}} g \\
+\frac{\nu \chi_{0}^{3}}{2 V \xi} e^{3 t^{2} / 2} \zeta=\frac{\chi_{0}^{6}}{4} e^{3 t^{2}} .
\end{gathered}
$$

Defining a new variable $v$ by $g=t e^{t^{2} / 4} v$, we get

$$
\begin{aligned}
v^{\prime \prime} & +\frac{1}{t} v^{\prime}+\left[\frac{32 \pi^{2} \chi_{0}^{4} e^{2 t^{2}}}{\nu^{2}}-\frac{1}{t^{2}}-\frac{t^{2}}{4}\right] v \\
& =\frac{8 \pi^{2} \chi_{0}^{6}}{\nu^{2} t} e^{11 t^{2} / 4}-\frac{16 \pi^{2} \chi_{0}^{3}}{\nu V \xi} e^{5 t^{2} / 4}\left(\frac{\zeta}{t}\right) .
\end{aligned}
$$

Again we decompose $v$ into a deterministic and a stochastic part, $v_{d}$ and $v_{s}$, respectively. The deterministic part admits a regular solution as $t \rightarrow 0$ :

$$
v_{d}=\frac{4 \pi^{2} \chi_{0}^{6}}{\nu^{2}} t \ln t+O\left(t^{3}\right) .
$$

In the same approximation, we find

$$
v_{s}=-\frac{8 \pi^{2} \chi_{0}^{3}}{\nu V \xi} \int_{t_{1}}^{t} d t^{\prime}\left[t-\frac{t^{\prime 2}}{t}\right]\left(\frac{\zeta}{t^{\prime}}\right)
$$

(more on $t_{1}$ later). Recalling that

$$
\left\langle\zeta(t) \zeta\left(t^{\prime}\right)\right\rangle=\frac{V}{192 \pi} \delta\left(\chi-\chi^{\prime}\right)=\frac{V}{192 \pi} \frac{e^{-t^{2} / 2}}{\chi_{0} t} \delta\left(t-t^{\prime}\right),
$$

we get, always in the same approximation, that 


$$
\left\langle v_{s}(t) v_{s}\left(t^{\prime}\right)\right\rangle=\left(\frac{8 \pi^{2} \chi_{0}^{3}}{\nu V \xi}\right)^{2} \frac{V}{192 \pi \chi^{0}} \int_{t_{1}}^{t} \frac{d s}{s^{3}}\left[t-\frac{s^{2}}{t}\right]\left[t^{\prime}-\frac{s^{2}}{t^{\prime}}\right],
$$

where $t_{<}=\min \left(t, t^{\prime}\right)$. Note that if we assume that the stochastic source acts all the way from $t=0$, we get an infinite amount of fluctuations.

As a matter of fact, we do not expect our equations to hold up to the smallest times. First of all, our semiclassical universe presumably arises out of a fully quantum one at the quantum to classical transition. The semiclassical equation thus describes the model up to this time, but not earlier. Besides, we do not expect the form (5.8) of the noise correlation function to be true up to this time. The actual noise self-correlation, which ought to be derived from quantum gravity, presumably has a finite correlation time (of the order of Planck's) and is not translation invariant, but depends on the distance from its arguments to the "absolute zero of time" (to borrrow Misner's phrase [51]). Equation (5.8) is thus an approximation to be trusted only when the characteristic times are much larger than Planck's. The lower cutoff $t_{1}$ can be thought of as the earliest time when the model becomes meaningful.

While we cannot make a definite prediction for $t_{1}$, we can still observe that if it is small enough, then fluctuations build up fast enough that they may actually dominate the deterministic part over an stretch of time, namely, when

$$
|\ln t| \leqslant \frac{1}{\sqrt{6 \pi}} \frac{\nu}{4 \xi V^{1 / 2} \chi_{0}^{7 / 2} t_{1}} .
$$

It is interesting to look at the behavior of a quantity of direct physical significance, such as the Hubble parameter $\mathcal{H}=\dot{a} / a^{2}$, rather than the metric itself. In terms of dimensionless quantities [cf. Eq. (3.11)] $\mathcal{H}=H / \sqrt{6} \ell_{P}$, where

$$
H=\frac{1}{b^{2}} \frac{d b}{d \chi} \text {. }
$$

Expanding $b(\chi)=\chi+\xi g(\chi)$ we can write $H$ in terms of $t$ $\left(\chi=\chi_{0} e^{t^{2} / 2}\right)$ and $v$ as

$$
H=\frac{1}{\chi^{2}}\left\{1+\frac{\xi}{\chi_{0}} e^{-t^{2} / 4}\left[\frac{d v}{d t}+\left(\frac{2-3 t^{2}}{2 t}\right) v\right]\right\} .
$$

Now, decomposing as above $H=H_{d}+H_{s}$, we obtain

$$
\begin{gathered}
H_{d} \sim \frac{1}{\chi^{2}}\left[1+\frac{4 \pi^{2} \xi \chi_{0}^{5}}{\nu^{2}}(2 \ln t+1)\right], \\
H_{s} \sim-\frac{16 \pi^{2} \chi_{0}^{2}}{\nu V \chi^{2}} \int_{t_{1}}^{t} d t^{\prime}\left(\frac{\zeta}{t^{\prime}}\right),
\end{gathered}
$$

which leads to

$$
\left\langle H_{s}^{2}\right\rangle=\left(\frac{16 \pi^{2} \chi_{0}^{2}}{\nu V \chi^{2}}\right)^{2} \frac{V}{384 \pi \chi^{0}}\left[\frac{1}{t_{1}^{2}}-\frac{1}{t^{2}}\right] .
$$

We see that, provided $t_{1}$ is small enough, the fluctuations may have an strong effect on the Hubble parameter.

\section{CONCLUSIONS}

In this paper we have considered the stochastic, classical metric fluctuations induced by quantum fluctuations of matter fields in two cases of interest; namely, the conformal fluctuations of flat spacetime and a simple cosmological model, the former being mostly of interest as preparation for the latter. The quantum fluctuations act on geometry through the energy momentum tensor, which has a deterministic part, associated to vacuum polarization and particle creation, and also a fluctuating part, related to the unpredictable aspects of quantum behavior; the introduction of the latter leads to the formulation of the theory in terms of a so-called EinsteinLangevin equation.

While the physical rationale for including such terms is clear (and earlier estimates point to their quantitative relevance $[5,6])$ and the technical means to introduce them consistently into Einstein equations are by now well established $[8,22,15,19,20]$ this is, to our knowledge, the first time actual nontrivial solutions to the Einstein-Langevin equation are obtained. The reason why the solution to particular models lagged so much behind the formulation of the basic principles lies of course in the staggering complexity of Einstein theory, involving issues of gauge invariance, renormalization and nonlinear dynamics. The model proposed by Hartle in Ref. [28] is an ideal testing ground because it retains most of the physical features of a realistic cosmological model, while being simple enough to be tractable.

Since in this last analysis massless nonconformal fields in FRW backgrounds are of interest mostly as a toy model for gravitons [29] (for the application of similar ideas to inflationary cosmology, see $[26,22-25])$, the effects we have discussed here are at bottom quantum gravitational, and they are most relevant in the earliest stages of cosmic evolution. This adds a degree of uncertainty in the theory, associated to the present lack of understanding of the details of the quantum to classical transition; the best we can do is to localize our ignorance in a few undetermined parameters, such as the time at which the initial conditions for semiclassical evolution are set and the value of the several renormalized parameters in the model (in particular, we have applied Occam's razor in setting to zero any parameter not specifically demanded by renormalization).

Given these limitations, the main conclusions of our effort are that the Einstein-Langevin equation may indeed be solved, that a consistent picture of semiclassical evolution emerges and, most important, that this picture is significatively different from what earlier (nonstochastic) semiclassical models have led us to believe (consider, for example, the ratio of the fluctuating to the deterministic parts of the Hubble parameter, as discussed in the last section). Clearly more work shall be needed before questions of direct cosmological impact (such as whether stochastic fluctuations may be instrumental in creating the homogeneous patches where inflation becomes a possibility, or rather work against the stability of those patches) may be addressed. We continue our research on this, in our opinion, most relevant issue of preinflation cosmology. 


\section{ACKNOWLEDGMENTS}

We are grateful to Bei-Lok Hu, Rosario Martín, Diego Mazzitelli, Juan Pablo Paz, and Josep Porrà for very helpful suggestions and discussions. This work has been partially supported by the European Project No. CI1-CT94-0004 and by the CICYT Contracts No. AEN95-0590 and AEN950882, Universidad de Buenos Aires, CONICET, and Fundación Antorchas.
[1] L. Rosenfeld, Nucl. Phys. B40, 353 (1963).

[2] N. D. Birrell and P. C. W. Davies, Quantum Fields in Curved Space (Cambridge University Press, Cambridge, England, 1982).

[3] J. P. Paz and S. Sinha, Phys. Rev. D 44, 1038 (1991); 45, 2823 (1992).

[4] M. Gell-Mann and J. B. Hartle, Phys. Rev. D 47, 3345 (1993).

[5] C.-I. Kuo and L. H. Ford, Phys. Rev. D 47, 4510 (1993).

[6] B. L. Hu and N. Phillips, Phys. Rev. D 55, 6123 (1997).

[7] E. Calzetta and B.-L. Hu, Phys. Rev. D 40, 656 (1989).

[8] E. Calzetta and B.-L. Hu, Phys. Rev. D 49, 6636 (1994).

[9] B.-L. Hu, Physica A 158, 399 (1989); in Quantum Classical Correspondence, edited by D. H. Feng and B. L. Hu (International Press, Boston, 1996); gr-qc/9607070.

[10] M. V. Fischetti, J. B. Hartle, and B.-L. Hu, Phys. Rev. D 20, 1757 (1979).

[11] J. B. Hartle and B.-L. Hu, Phys. Rev. D 20, 1772 (1979); 21, 2756 (1980); 22, 2091 (1980).

[12] E. Calzetta and B.-L. Hu, Phys. Rev. D 35, 495 (1987).

[13] E. Calzetta, Class. Quantum Grav. 6, L227 (1989); Phys. Rev. D 43, 2498 (1991); E. Calzetta, M. Castagnino, and R. Scoccimarro, ibid. 45, 2806 (1992).

[14] H. Callen and T. Welton, Phys. Rev. 83, 34 (1951); L. Landau, E. Lifshitz, and L. Pitaevsky, Statistical Physics (Pergamon, London, 1980), Vol. 1; S. K. Ma, Modern Theory of Critical Phenomena (Benjamin, London, 1976); J. P. Boon and S. Yip, Molecular Hydrodynamics (Dover, New York, 1991).

[15] B.-L. Hu and S. Sinha, Phys. Rev. D 51, 1587 (1995); B.-L. $\mathrm{Hu}$ and A. Matacz, ibid. 51, 1577 (1995).

[16] L. Parker, Phys. Rev. Lett. 21, 562 (1968); Phys. Rev. 183, 1057 (1969).

[17] Y. B. Zeldovich, Pis'ma Zh. Éksp. Teor. Fiz. 12, 443 (1970) [JETP Lett. 12, 307 (1970)].

[18] B. L. Hu, J. P. Paz, and Y. Zhang, Phys. Rev. D 45, 2843 (1992); 47, 1576 (1993).

[19] A. Campos and E. Verdaguer, Phys. Rev. D 53, 1927 (1996).

[20] F. Lombardo and F. D. Mazzitelli, Phys. Rev. D 55, 3889 (1997).

[21] R. P. Feynman and F. L. Vernon, Ann. Phys. (N.Y.) 24, 118 (1963); R. P. Feynman and A. R. Hibbs, Quantum Mechanics and Path Integrals (McGraw-Hill, New York, 1965).

[22] E. Calzetta and B.-L. Hu, Phys. Rev. D 52, 6770 (1995).

[23] E. Calzetta and S. Gonorazky, Phys. Rev. D 55, 1812 (1997).

[24] A. Matacz, Phys. Rev. D 55, 1860 (1997).

[25] A. Matacz, gr-qc/9611063.
[26] M. Morikawa, Vistas Astron. 37, 87 (1993).

[27] E. Calzetta and B.-L. Hu, Phys. Rev. D 55, 3536 (1997).

[28] J. B. Hartle, Phys. Rev. D 23, 2121 (1981).

[29] L. P. Grishchuk, Zh. Éksp. Teor. Fiz. 67, 825 (1974) [Sov. Phys. JETP 40, 409 (1975)].

[30] G. T. Horowitz, Phys. Rev. D 21, 1445 (1980).

[31] E. E. Flanagan and R. M. Wald, Phys. Rev. D 54, 6233 (1996).

[32] J. B. Hartle, Phys. Rev. Lett. 39, 1373 (1977).

[33] A. A. Starobinsky and Y. B. Zeldovich, Zh. Éksp. Teor. Fiz. 61, 2161 (1971) [Sov. Phys. JETP 34, 1159 (1972)]; Pis'ma Zh. Éksp. Teor. Fiz. 26, 377 (1977) [JETP Lett. 26, 256 (1977)].

[34] N. D. Birrell and P. C. W. Davies, J. Phys. A 13, 2109 (1980).

[35] V. N. Lukash and A. A. Starobinsky, Zh. Éksp. Teor. Fiz. 66, 1515 (1974) [Sov. Phys. JETP 39, 742 (1974)]; V. N. Lukash, I. D. Novikov, A. A. Starobinsky, and Y. B. Zeldovich, Nuovo Cimento B 35, 293 (1976); B.-L. Hu and L. Parker, Phys. Rev. D 17, 933 (1978).

[36] J. A. Frieman, Phys. Rev. D 39, 389 (1989); J. Céspedes and E. Verdaguer, ibid. 41, 1022 (1990); A. Campos and E. Verdaguer, ibid. 45, 4428 (1992).

[37] A. Campos and E. Verdaguer, Phys. Rev. D 49, 1861 (1994).

[38] A. A. Starobinsky, Phys. Lett. 91B, 99 (1980).

[39] P. Anderson, Phys. Rev. D 28, 281 (1983); 29, 615 (1984).

[40] R. Wald, Quantum Field Theory in Curved Spacetime and Black Hole Thermodynamics (The University of Chicago Press, Chicago, 1994).

[41] J. Z. Simon, Phys. Rev. D 43, 3308 (1991); L. Parker and J. Z. Simon, ibid. 47, 1339 (1993).

[42] P. Anderson, Phys. Rev. D 32, 1302 (1985); 33, 1567 (1986).

[43] J. Schwinger, J. Math. Phys. (N.Y.) 2, 407 (1961); Phys. Rev. 128, 2425 (1962); P. M. Bakshi and K. T. Mahanthappa, J. Math. Phys. (N.Y.) 4, 1 (1963); 4, 12 (1963).

[44] L. V. Keldysh, Zh. Éksp. Teor. Fiz. 47, 1515 (1964) [Sov. Phys. JETP 20, 1018 (1965)].

[45] K. Chou, Z. Su, B. Hao, and L. Yu, Phys. Rep. 118, 1 (1985).

[46] R. D. Jordan, Phys. Rev. D 33, 444 (1986); 36, 3593 (1987).

[47] E. S. Abers and B. W. Lee, Phys. Rep., Phys. Lett. 9C, 1 (1973).

[48] A. Campos and E. Verdaguer, Int. J. Mod. Phys. (to be published).

[49] L. Kadanoff and G. Baym, Quantum Statistical Mechanics (Benjamin, New York, 1962).

[50] E. Calzetta and B.-L. Hu, Phys. Rev. D 37, 2878 (1988).

[51] C. Misner, Phys. Rev. 186, 1328 (1969). 